\section{Hints of hope with remdesivir}

The FDA issued an emergency use authorization (EUA) for Gilead's remdesivir for COVID-19 in May, even as it awaits more concrete evidence of benefit.

Gilead's remdesivir is a direct-acting antiviral drug that inhibits viral RNA polymerases, blocking RNA synthesis. First advanced as a potential treatment of Ebola, where it failed in the clinic, it has preclinical activity against other viruses including SARS-CoV-2. As such, it was one of the early drugs moved into the clinic as a possible treatment for COVID-19.

Clinical data supporting use of the drug, however, remain mixed.

In a first randomized trial of the drug, investigators in China recruited 237 patients with severe COVID-19 onto treatment or placebo. The drug failed to meet its primary end point - time to clinical improvement - the investigators reported in The Lancet in April. This trial was stopped early, however, owing to declining patient numbers in China. In an associated comment in The Lancet, medical statistician John Norrie, of Edinburgh University, cautioned that the underpowered trial consequently sheds little light on the efficacy of the drug. "The study has not shown a statistically significant finding that confirms a remdesivir treatment benefit of

\section{COVID umbrella trials multiply}

Several master protocols that can test multiple drugs in parallel are now underway in COVID-19. By streamlining patient recruitment, standards of care, data collection and more, these could generate more robust data than stand-alone trials, and enable more efficient evaluation of the proposed drug pipeline.

For now, these trials are still testing repurposed agents, primarily in hospitalized patients with confirmed COVID-19.

The UK's 9,600-patient RECOVERY trial, for instance, is randomizing patients into five arms: the HIV drugs lopinavir-ritonavir; the steroid dexamethasone; the antimalarial hydroxychloroquine; the antibiotic azithromycin; and the anti-inflammatory IL-6 blocker tocilizumab.

The WHO's SOLIDARITY trial is set up such that countries around the world can participate, regardless of their infrastructural capabilities. It had recruited 1,200 patients by 21 April. Treatment arms consist of Gilead's RNA-polymerase inhibitor remdesivir, chloroquine or hydroxychloroquine, lopinavirritonavir, and lopinavir-ritonavir plus the immune-modulatory agent IFN $\beta_{1 \text { a. }}$. at least the minimally clinically important difference, nor has it ruled such a benefit out," he wrote.

Top-line data from the NIAID-sponsored ACTT trial are more encouraging. Preliminary results disclosed in an NIAID press release suggest that remdesivir-treated patients recovered $31 \%$ faster than those who received placebo (11 days compared with 15 days). The NIAID has not yet published the detailed results of this trial.

Gilead has published a summary of compassionate use data, from 61 patients with severe COVID-19. Clinical improvement was observed in $68 \%$ of patients, they reported in the New England Journal of Medicine. But this was not a controlled trial, and these differences could be due to differences in supportive care, in institutional treatment protocols or in hospitalization thresholds, the authors acknowledge.

The FDA issued its EUA "based on the totality of scientific evidence" and the absence of alternative options.

Other trials that could provide more definitive data are ongoing, including a 6,000-patient trial from Gilead and the WHO's SOLIDARITY umbrella trial. Asher Mullard

The NIAID's ACTT trial enrolled over 1,000 hospitalized patients with COVID-19 onto remdesivir or placebo. Preliminary results from this trial prompted the FDA to issue an Emergency Use Authorization for remdesivir. A follow-on 1,000-patient trial called ACTT 2 is now testing Lilly's anti-inflammatory JAK inhibitor baricitinib in combination with remdesivir, versus placebo plus remdesivir.

But agents also need to be tested earlier in the course of disease. The UK's 3,000-patient PRINCIPLE trial is enrolling high-risk and older patients with symptoms of disease to reduce the need for hospitalization. A first arm is testing hydroxychloroquine, and a second will test azithromycin.

REMAP-CAP, first set up for communityacquired pneumonia, has added a COVID-19 component. To date, 420 patients have been recruited. In addition to hydroxychloroquine, lopinavir-ritonavir, IFN $\beta$, corticosteroid strategies and macrolide therapy, it is also being amended to evaluate the IL-1 receptor antagonist anakinra and the IL- 6 blockers tocilizumab and sarilumab.

With the COVID-19 pipeline growing, there is also a need for trials that can generate preliminary data to prioritize follow-on work. The UK's 1,800-patient ACCORD trial in hospitalized patients with COVID-19 is taking this on, setting out to test six agents that could then be advanced into larger umbrella trials. As Nature Reviews Drug Discovery went to press, disclosed agents in this trial included BerGenBio's AXL inhibitor bemcentinib, AstraZeneca's IL-33 mAb MEDI-3506 and AstraZeneca's BTK inhibitor acalabrutinib.

The NIH has also setup ACTIV, to prioritize vaccine and drug candidates for clinical trials.

Over 115 vaccine candidates have also been proposed. The $\mathrm{WHO}$ is working on a master protocol for the most promising vaccines.

Asher Mullard

\section{Parkinson disease setback}

Roche and partner Prothena's antibody to a-synuclein, prasinezumab, has failed in a first phase II trial in Parkinson disease.

$\alpha$-Synuclein is a presynaptic neuronal protein that is linked to Parkinson disease. Pathologically, misfolded $\alpha$-synuclein monomers form oligomers and fibrils, which can aggregate into the protein inclusions called Lewy bodies that typify Parkinson disease brains. A missense mutation in the $\alpha$-synuclein gene, SNCA, was also the first specific genetic aberration linked to the disease. As such, it has caught the attention of drug developers. Roche and Prothena have been leading the way with prasinezumab, an antibody that binds toxic forms of $\alpha$-synuclein.

In 2017, the partners launched a 316-patient, placebo-controlled trial of the antibody, looking for a difference on the Movement Disorder Society-Unified Parkinson's Disease Rating Scale (MDS-UPDRS) scale at 52 weeks. The study did not meet this primary end point, the companies have now reported. A blinded extension study is ongoing. An update on the antibody is due later this year.

Other companies are also going after $\alpha$-synuclein. Biogen, AbbVie and Lundbeck/Genmab and AstraZeneca/Takeda all have antibodies to $\alpha$-synuclein in the clinic. Affiris and United Neuroscience have therapeutic vaccines in development. And UCB/Neuropore are testing a small-molecule inhibitor of $\alpha$-synuclein misfolding.

Questions for these companies include which forms of $\alpha$-synuclein to target, whether candidates penetrate sufficiently into the brain and how early you have to intervene to see efficacy - reminiscent of the situation with amyloid-modulating and tau-modulating agents in Alzheimer disease.

Asher Mullard 